\title{
Caracterización florística y estructural de especies arbóreas asociadas al bosque de encino-pino de la Reserva Santa Rosa, Estelí, Nicaragua
}

\author{
Floristic and structural characterization of tree species associated with the pine-oak forest of the Santa Rosa \\ Reserve, Estelí, Nicaragua
}

\author{
Luz Esmelda Reynoza Ruiz ${ }^{1}$, Enrique Mayorga Arostegui ${ }^{2}$ \\ ${ }^{1}$ MSc. en Gestión Ambiental / ORCID: https://orcid.org/0000-0003-4553-5933 / lereynosa@ci.una.edu.ni \\ ${ }^{2}$ Ingeniero en Recursos Naturales Renovables / ORCID: https://orcid.org/0000-0002-3473-039X / enrique.mayorga@ci.una.edu.ni \\ Universidad Nacional Agraria, Facultad de Recursos Naturales y el Ambiente
}

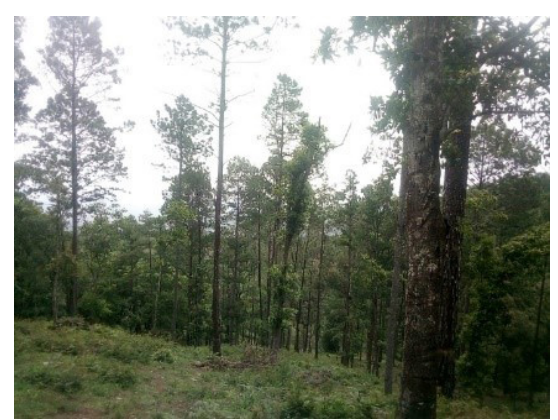

\section{RESUMEN}

La caracterización estructural es uno de los principales fundamentos para entender la distribución y dominancia espacial de las especies forestales. El estudio se desarrolló en la Reserva Santa Rosa ubicado en el municipio de Estelí en la comunidad Almaciguera. Se realizó el levantamiento de cuatro parcelas circulares de $314 \mathrm{~m}^{2}$ cada una, a lo largo de un gradiente florístico de $1 \mathrm{~km}$. Se registró el diámetro, altura, edad de los árboles y se recolectó material vegetativo a nivel herbáceo y arbustivo para caracterizar las estructuras de las especies en la asociación encontrada. Las especies reportadas en las parcelas mostró un total de 13 familias, 14 géneros y 19 especies herbáceas y arbustivas; se identificó especies bajo el dosel del bosque, especies herbáceas y arbustivas generalistas y selectivas de acuerdo a gradientes ambientales. La composición florística reportó densidades similares para $Q$. sapotifolia y densidades variantes para $P$. maximinoi. La prueba Kruskal Wallis reflejó una $\mathrm{p}=0.416$ en cuatro rangos diferentes, los datos no presentaron diferencias significativas en la asociación. El coeficiente de Gini presentó los valores más desiguales para la parcela dos del área intervenida con 0.86 , además de presentar la variación mayor en relación al diámetro con $79 \mathrm{~cm}$ para Quercus y presentar las parcelas más diversas en cuanto al número de especies. La dominancia, estuvo representada por Pinus con $15.61 \mathrm{~m}^{2} \mathrm{ha}^{-1}$. El IR lo registró Pinus con $139.57 \%$ en la parcela dos del área de bosque de pino esto se debe a que existe una etapa sucesional inicial y/o intermedia, debido al proceso de regeneración natural que ha presentado la especies como una estrategia para su permanencia.

Palabras clave: índice de rendimiento, coeficiente de Gini, densidad, dominancia.

\begin{abstract}
Structural characterization is one of the main foundations for understanding the spatial distribution and dominance of forest species. The studio was developed in the Reserva Santa Rosa located in the municipality of Estelí in the Almaciguera community. Four circular plots of $314 \mathrm{~m}^{2}$ each were lifted along a floristic gradient of $1 \mathrm{~km}$. The diameter, height, age of the trees and collected was recorded. herbaceous and shrub-level vegetative samples to characterize species structures in the association found. The species reported on the plots showed a total of 13 families, 14 genera and 19 herbaceous and shrub species; considering data on structural variations. The floristic composition reported similar densities for $Q$. sapotifolia and variant densities for P. maximinoi. The Kruskal Wallis test reflected a $\mathrm{p}=0.416$ in three different ranges despite this, the data did not have significant differences in the association. The Gini coefficient presented the most unequal values for plot two of the intervened area with 0.86 , in addition to presenting the largest variation in relation to the diameter with $79 \mathrm{~cm}$ for Quercus and presenting the most diverse plots in terms of the number of species. The dominance was represented by Pinus at $15.61 \mathrm{~m}^{2} \mathrm{ha}^{-1}$. The IR was recorded by Pinus with $139.57 \%$ on plot two of the pine forest area this is because there is an initial and/or intermediate succession stage, due to the natural regeneration process.
\end{abstract}

Keywords: Performance of index, Gini coefficient, density, dominance.
Recibido: 30 de enero del 2020

Aceptado: 10 de octubre del 2020
Los artículos de la revista La Calera de la Universidad Nacional Agraria, Nicaragua, se comparten bajo términos de la licencia Creative Commons: Reconocimiento, No Comercial, Compartir Igual. Las autorizaciones adicionales a las aquí delimitadas se

C copyright 2020. Universidad Nacional Agraria (UNA).jimenez@ci.una.edu.ni 


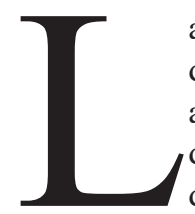

a estructura de un sitio forestal es el resultado de los hábitos de crecimiento de las especies arbóreas, de las condiciones ambientales y de las prácticas de manejo bajo las cuales se desarrolló y originó el sitio a través del tiempo (Wenger, 1984; Olvera-Vargas y Figueroa-Rangel, 2012).

La ecología vegetal presenta muchas interrogantes para los diferentes estudios en la dinámica del bosque. Comprender estas interrogantes permiten que muchas de las plantas puedan sobrevivir y crecer bajo condiciones ambientales que identifiquen los mecanismos que controlan estos patrones de distribución (Galindo-Jaimes, 2002).

La caracterización estructural se constituye en uno de los fundamentos principales para el entendimiento de la distribución y dominancia espacial de las especies forestales. La variación estructural de las poblaciones forestales depende en gran medida de la composición de especies de las comunidades, de tal forma que una misma especie puede presentar condición estructural de acuerdo con la asociación florística donde se encuentra coexistiendo (Johnson et al., 2002; Olvera-Vargas y Figueroa-Rangel, 2012).

Aunque la diversidad de especies podría considerarse baja localmente en el ecosistema de pinoencino (Pinus-Quercus), se presenta una gran diversidad en los estratos herbáceos y arbustivos. Por otra parte, a nivel de paisaje existe gran variación altitudinal dentro de áreas relativamente cercanas, con diferencias en la inclinación y la orientación de las laderas, el tipo y profundidad del suelo y la compleja historia geotectónica (Challenger y Caballero, 1998; Siles et al., 2017). Dentro de la eco-región del bosque de pino-encino en Nicaragua, existen diferentes áreas protegidas dentro de las que se encuentra la Reserva Natural Tisey-Estanzuela con una extensión de 9500 ha y a una altitud entre 700 y 1500 msnm (Cornide-Rivas et al., 2008).

Para comprender la finalidad del estudio (Kappelle, 2006; Luna-Vega et al., 2006) sugieren que el conocimiento de la estructura de los bosques dominados por encino, así como su relación con el ambiente donde se desarrollan, es un tema fundamental para sustentar el diseño de estrategias de conservación y manejo a largo plazo de estos ecosistemas. En este sentido, los objetivos fueron: i) caracterizar la estructura de las especies presentes en asociaciones de Pinus-Quercus, ii) Comparar sus cambios y diferencias estructurales a través de variables ambientales y datos dasométricos y iii) Deducir la relación de cambios ambientales con énfasis en la vegetación a través de indicadores de interés forestal.

\section{MATERIALES Y MÉTODOS}

Localización del área de estudio. El presente estudio se llevó a cabo en la Reserva privada Santa Rosa (propiedad de Eriberto Cerrato), ubicada en el área protegida de TiseyEstanzuela (latitud Norte $12^{\circ} 55^{\prime}$ y longitud Oeste $86^{\circ} 21$ ) en el departamento de Estelí, Nicaragua. El área protegida está clasificada como Reserva Natural según la Ley General de Medio Ambiente y Recursos Naturales de Nicaragua de acuerdo con el Ministerio de Recursos Naturales y del Ambiente (MARENA, 2007). Se encuentra ubicada en las cordilleras centrales y se caracterizan por terrenos con relieves muy accidentados, su punto más alto es el Cerro Tisey a $1500 \mathrm{msnm}$. La temperatura anual presenta valores menores a $24{ }^{\circ} \mathrm{C}$ y precipitación anual entre los 1000 y 1 $200 \mathrm{msnm}$.

Registro de información y análisis estadístico. La información recopilada en este estudio se derivó del establecimiento de cuatro parcelas circulares de $314 \mathrm{~m}^{2}$, establecidas en bosques de pino dominado por encino a lo largo de un gradiente florístico de $1 \mathrm{~km}$ de longitud y un rango altitudinal de 700 a $1500 \mathrm{msnm}$.

En cada parcela se registró el nombre de la especie, diámetro a la altura del pecho (DAP) y altura total para todos los individuos con DAP $>10 \mathrm{~cm}$.

Además de la información dasométrica de las especies en cada una de las parcelas, se hizo la identificación de especies herbáceas y arbustivas en campo ayudados con guías dendrológicas (Gentry, 1993; Holdridge y Poveda, 1997; Jardim et al., 2003; Keller, 2004; Killen et al., 1998; Ulloa y Jorgensen, 1993; Zamora, 2000; Zamora et al. 2004; Zamora et al. 2000; Zamora y Pennington, 2001).

Aquellas especies que no fueron identificadas se colectaron con materia fértil para ser identificadas posteriormente en el herbario de la Universidad Nacional Agraria y con la ayuda de un experto en la identificación de especies forestales.

Para caracterizar la condición estructural de cada asociación florística presente en las parcelas de estudio, se estimó la densidad total por hectárea y el área basal total por hectárea.

La comparación estructural se realizó a partir del método no paramétrico de Kruskal-Wallis con el programa estadístico Infostat 2013, haciendo uso de la frecuencia de individuos por clase diamétrica para determinar diferencias entre los tamaños diamétricos entre las especies arbóreas presentes en las parcelas, y así determinar las densidades más sobresalientes en las asociaciones presentadas en las cuatro parcelas de Quercus-Pinus.

Así mismo, se evalúo la igualdad de las distribuciones diamétricas de las especies por asociación florística; se utilizó el coeficiente de Gini (G); G es un índice 
representa igualdad perfecta y 1 desigualdad total. Se calculó el $\mathrm{G}$ utilizando los datos individuales de cada especie por asociación según Dixon (2001).

$$
G=\frac{\sum_{i=1}^{n}(2 i-n-1) X i}{(n-1) \sum_{i=1}^{n} X i}
$$

Dónde: $\mathrm{n}=$ número de individuos en la muestra; $\mathrm{X}_{i}$ es el tamaño (diámetro) del individuo ith, ordenado del menor al mayor, $\mathrm{X}_{i} \leq \mathrm{X}_{2} \leq \ldots \leq \mathrm{X}_{\mathrm{n}} ; i$ representa el diámetro individual de cada árbol.

Por otra parte, para conocer las similitudes de sitio en cuanto a las especies herbáceas y arbustivas en las áreas del bosque y área intervenida de Quercus-Pino, fue mediante un análisis de conglomerados con el programa de cómputo Infostat 2013, tomando en cuenta las familias reportadas y lo sitios donde se establecieron las parcelas de estudio.

Para el análisis de los cambios ambientales se utilizó el índice de rendimiento (IR) de todos los individuos de cada especie presente en cada asociación florística.

Según Olvera-Vargas et al. (2010) el índice de rendimiento (IR) es un estimador integrado por la densidad relativa y el volumen relativo de cada especie:

$$
\begin{gathered}
\mathrm{IR}=\text { Densidad Relativa }+ \text { Volumen Relativo } \\
\text { Dónde: } \\
\text { Densidad Relativa }=\left(\frac{D S p i}{\sum D S p i}\right) * 100 \\
\text { Volumen Relativo }=\left(\frac{\text { Volspi }}{\sum \text { Volspi }}\right) * 100
\end{gathered}
$$

DSpi es el número de individuos de la especie $i$ en la parcela $j$; VolSpi es el volumen de la especie $i$ en la parcela j. El volumen se calculó de acuerdo a Husch et al. (1982):

$$
\begin{gathered}
\operatorname{Vol}=(\bar{h})(G)(f) \mid \\
\bar{h}=\frac{n_{1} g_{1} h_{1}+n_{2} g_{2} h_{2}+\cdots \cdot n_{z} g_{z} h_{z}}{G}=\frac{\sum_{i=1}^{z} n_{i} g_{i} h_{i}}{\sum_{i=1}^{z} n_{i} g_{i}}
\end{gathered}
$$

Dónde: $\mathrm{n}_{i}$ es el número de individuos por especie por clase diamétrica, $\mathrm{g}_{i}$ es el área basal media por especie por clase diamétrica, y hi es la altura media por especie y clase diamétrica; $\mathrm{G}$ es el área basal total por parcela; $f$ es el factor de forma que se definió con un valor de 0.5 para todas las especies. 0.5 se considera como un valor estandarizado para aquellas especies para las cuales el valor específico es desconocido (Husch et al., 1982).

\section{RESULTADOS Y DISCUSIÓN}

Caracterización florística y estructural. Se registraron 13 familias, representadas en 14 géneros y 19 especies arbóreas en conjunto con especies a nivel herbáceo y arbustivo. Las parcelas establecidas reportaron un total de 41 individuos a nivel arbóreo oscilando diámetros entre 10 a $79 \mathrm{~cm}$ respectivamente.

Las familias con mayor número de especies fueron Mimosaceae y Fabaceae (dos géneros y dos especies). Las especies encontradas con mayor abundancia fueron: Rubus urticifolius, Pteridium arachnoideum, y Desmodium sp. Por el contrario, nueve especies presentaron una baja abundancia siendo algunas de ellas Bouvardia multiflora Schutt, Capraria biflora, Monotropa uniflora y Boerhavia erecta L.

Encina et al. (2009); Rubio-Licona et al., (2011); Rocha-Loredo et al. (2010) y Morrone, (2005), reportan estudios sobre hábitats en bosques de encino-pino, cuyas familias que coexisten en el dosel medio y bajo de este ecosistema forestal en México, algunas de estas familias presentan una similitud en cuanto a su composición florística en el presente estudio como Fabaceae, Rosaceae, Mimosaceae y Fagaceae.

La diferenciación florística encontrada en las parcelas de estudios se reflejó en dos especies Quercus y Pinus a nivel arbóreo, mientras que a niveles más bajo del dosel se reportan alrededor de 11 géneros, siendo alguno de ellos: Sauraraia, Miconia, Lantana, Mimosa, Rubus y Capraria. Rzedowski (1996), reporta en sus estudios géneros como Quercus, Pinus, Ocotea, Clethra, Sauraria, Miconia, Inga, Eugenia y Arachnothryx. Sin embargo, en este estudio solo se registró Sauraria y Miconia, como una similitud en cuanto a su composición, otros géneros tales como Pteridium, Miconia, Rubus y Vachellia fueron especies más generalistas del sitio, al encontrarse en las cuatro parcelas de estudios.

Otros géneros como Mimosa, Lantana, Piper, Bouvardia, Stigmaphyllon, Capraria, Sauraria y Miconia, fueron especies comúnmente encontrada en las partes del área de bosque intervenido. Una particularidad del género Monotropa, es que solo se reportó en una parcela del estudio, siendo una especie que debido a sus condiciones y requerimientos se ubican debajo del dosel de los árboles para su óptimo desarrollo, cada una de las especies reportadas han sido o están fuertemente influenciadas por los gradientes ambientales.

En la Reserva Natural Tisey-Estanzuela en las zonas bajas menores a los $800 \mathrm{msnm}$ es posible encontrar bosque seco caducifolio (Siles et al., 2017) mientras que, a altitudes mayores a $1300 \mathrm{msnm}$, se pueden encontrar bosques dominados por $Q$. segoviensis; en las parcelas establecidas en estudio entre los 1000 y $1200 \mathrm{msnm}$, se presentan bosques dominados por $Q$. sapotifolia en un asociación con P. maximinoi, donde coexisten bajo el dosel una diversidad de especies herbáceas y arbustivas perennes y estacionales que están influenciadas por la altitud, luz solar y precipitación principalmente .

En cuanto a las variaciones estructurales de la composición florística, se encontró que la abundancia de las especies, medida a través de su densidad fue variante, siendo Quercus sapotifolia la que presenta una densidad similar en cada una de las parcelas, esta tendencia pueda deberse a que la mayoría de las parcelas presenta un dominio de Quercus, reflejando la necesidad de mantener una regeneración 
estratégica y competir por los recursos necesarios para su desarrollo; en cambio Pinus maximinoi presentó una densidad muy variante, este comportamiento puede deberse a que en la zona de estudio se están realizando aprovechamiento forestal de esta especies, si se observa en el Cuadro 1, los valores más bajo de densidad se sitúa en la parcela 2 del área de bosque, dicha parcela presenta un dominio del $60 \%$ del Quercus con respecto al Pinus, las densidades más altas se alcanzan en las parcelas del bosque intervenido, donde se han aplicado tratamientos silviculturales para el manejo forestal del Pinus.

En cuanto a la dominancia, evaluada por medio del área basal en las cuatro parcelas se reportó que Pinus maximinoi representó la mayor área basal con $15.61 \mathrm{~m}^{2} \mathrm{ha}^{-1}$ en el área de bosque, ya que la mayoría de estas especies presentan un diámetro promedio de $45 \mathrm{y} 79 \mathrm{~cm}$ respectivamente, mientras que la menor densidad se reportó en el área de bosque intervenida en Quercus sapotifolia con un valor de $6.37 \mathrm{~m}^{2} \mathrm{ha}^{-1}$ con un valor promedio en diámetro entre los 23 y $48 \mathrm{~cm}$ respectivamente (Cuadro 1 y 4).

Cuadro 1. Área basal y densidad por hectárea de las parcelas en estudio, Reserva Santa Rosa, Estelí

\begin{tabular}{lcccccccc}
\hline Especie & \multicolumn{3}{c}{$\mathrm{G}\left(\mathrm{m}^{2} \mathrm{ha}^{-1}\right)$} & \multicolumn{4}{c}{ Densidad $\left(\mathrm{Ind} \mathrm{ha}{ }^{-1}\right)$} \\
\hline Parcela & $1(\mathrm{~B})$ & $2(\mathrm{~B})$ & $1(\mathrm{In})$ & $2(\mathrm{In})$ & $1(\mathrm{~B})$ & $2(\mathrm{~B})$ & $1(\mathrm{In})$ & $2(\mathrm{In})$ \\
Q. sapotifolia & 1.27 & 2.87 & 6.37 & 1.59 & 159 & 159 & 159 & 191 \\
P. maximinoi & 5.73 & 15.61 & 7.01 & 2.87 & 127 & 32 & 191 & 287 \\
\hline
\end{tabular}

G: Área basal, $\mathrm{m}^{2} \mathrm{ha}^{-1}$ : metros cuadrados por ha, Ind ha ${ }^{-1}$ : Individuos por ha.

Comparaciones estructurales a partir de variables ambientales y datos dasométricos. Los resultados de la prueba no paramétrica de Kruskal-Wallis mostró cuatro rangos en cuanto a la frecuencia diamétrica (Figura 1) y las densidades más sobresalientes (Cuadro 1); los datos obtenidos con relación a $\mathrm{p}=0.416$ (ns), sugieren de acuerdo a la interacción con las variables estudiadas, que no presentan diferencias significativas en cuanto a su forma de distribución y tamaño diamétrico y a su vez a su abundancia que fue medida por medio de la densidad por hectárea en la asociación de Quercus-Pinus en el área de bosque y el área intervenida (Cuadro 2).

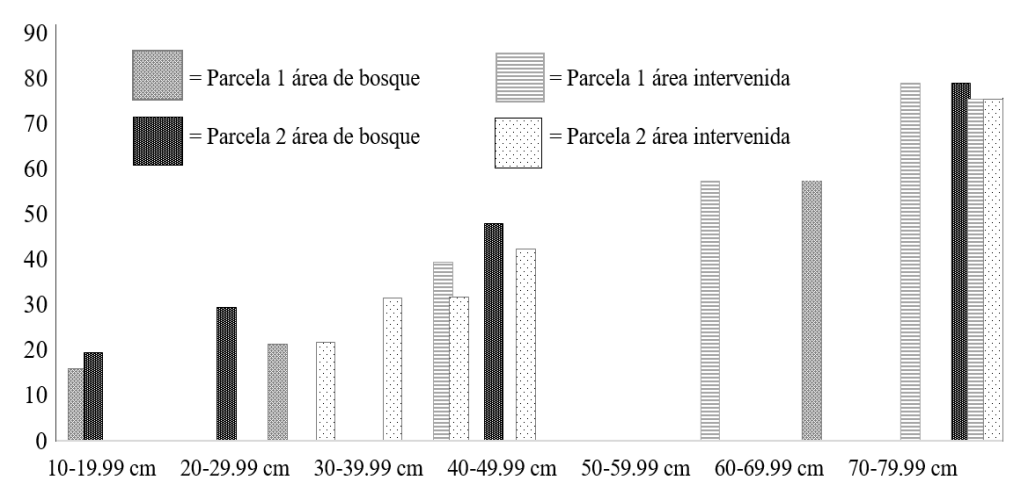

Figura 1. Distribución de la frecuencia diamétrica en la Reserva Santa Rosa, Estelí. y Boraginaceae.
Cuadro 2. Estadística de prueba Kruskal Wallis, Reserva Santa Rosa, Estelí

\begin{tabular}{cc}
\hline \multicolumn{2}{c}{ Estadísticos de prueba ${ }^{\text {a }, \mathbf{b}}$} \\
\hline & $\begin{array}{c}\text { Densidad } \\
\text { sobresaliente }\end{array}$ \\
\cline { 2 - 2 } H de Kruskal-Wallis & 5.000 \\
gl & 5 \\
Sig. asintótica & $0.416(\mathrm{~ns})$ \\
\hline
\end{tabular}

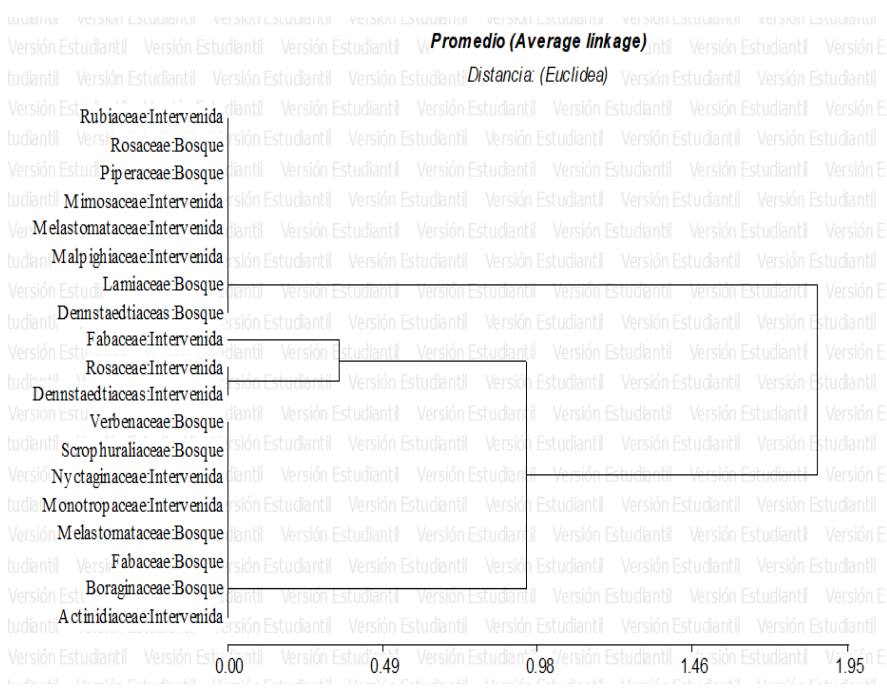

Figura 2. Prueba de conglomerados de las asociaciones en las parcelas de muestreo, Reserva Santa Rosa.

Se analizó a partir de una prueba de conglomerados, donde hay similitudes presentadas en cada una de las parcelas. La prueba mostró que en la Reserva Santa Rosa se presentaron dos asociaciones de vegetación con base en la dominancia de Quercus. Todas las asociaciones mostraron un comportamiento de asociación arbóreo entre Pino y Roble. Por otro parte Koleff y Urquiza-Hass, (2011); Cornide et al. (2008) y Rocha-Loredo et al. (2010) mencionan que los bosques de pinos dominados por encinos son en realidad un conjunto de diversas asociaciones de vegetación, dichas asociaciones se mostró en el presente en el estudio a nivel del dosel bajo del bosque, encontrándose entre los sitios de muestreo las siguientes familias: Rosaceae, Melastomatacea, Fabaceae, y Dennstaedtiaceas encontradas más recurrentes en el comportamiento asocio arbóreo del bosque encino-pino (Figura 2).

La familia Dennstaedtiaceas fue la familia más generalista de todas las especies reportadas ya que sus requerimientos en hábitats son bastantes extensos (bosques húmedos, abiertos, pendientes, otros), otras por su parte fueron más selectivas al solo presentarse en las áreas donde no existen aprovechamientos forestales tales como Piperaceae, Scrophuraliaceae, Lamiaceae 
Los resultados del coeficiente de Gini indican que en las cuatro parcelas se presentan valores diferenciados, la mayor desigualdad la presentó la parcela dos del área intervenida con 0.86 , esta desigualdad se asume como una respuesta diferencial de las especies presentes en esta asociación, en particular la especie de Pinus, que efectúan procesos de competencia con mayor número de especies (nueve individuos reportadas en la parcela).y la igualdad más cercana se registra en la parcela dos del área de bosque con 0.6 , en particular en esta parcela de estudio se presentan los valores más bajo en cuanto al número de individuos (seis individuos) (Cuadro 3).

Cuadro 3. Coeficiente de Gini por parcelas, Reserva Santa Rosa, Estelí

\begin{tabular}{lc}
\multicolumn{1}{c}{ Parcelas } & $\mathrm{G}$ \\
\hline Parcela 1 Bosque & 0.75 \\
Parcela 2 Bosque & 0.60 \\
Parcela 3 Intervenida & 0.80 \\
Parcela 4 Intervenida & 0.86 \\
\hline G: Coeficiente de Gini. &
\end{tabular}

Olvera-Vargas et al. (2015), mostraron resultados del Coeficiente de Gini para bosques de Quercus en asocio con Pinus entre rangos de 0.35 a 0.43 , y establecieron diámetros relativamente uniformes, en los periodos de recolección de datos y se presentaron pequeñas diferencias de tamaños tales como $Q$. castanea y Q. rugosa al presentar las mayores desigualdades.

Olvera-Vargas y Figueroa-Rangel, (2012) indica de igual forma que dependiendo de la asociación donde se encuentre las especies, pueden existir desigualdad en los tamaños diamétricos entre sus individuos y reporta que $Q$. crassipes de la asociación 2 presentó la mayor desigualdad de tamaños diamétricos $(\mathrm{G}=0.55)$ de todas las especies. Esta desigualdad en los tamaños diamétricos es un efecto asociado a procesos competitivos que llevan a cabo las especies durante las diferentes etapas de desarrollo del rodal.

De acuerdo con Metsaranta y Lieffers (2008) un valor de $\mathrm{G}<0.5$ indica que el tamaño de todos los individuos en la población es el mismo. Consecuentemente, lacompetencia entrelosindividuos de la población es simétrica entre todos los tamaños. En el caso de los estudios reportados como en México, se indican tamaños similares y algunos con variaciones en cuanto a su distribución en diámetros para competir por recursos, por otra parte, en la Reserva Santa Rosa, los valores en cuanto al índice de Gini, reporto valores arriba por $\mathrm{G}=0.5$, esto indica que los diámetros difieren gradualmente a su respuesta para obtener espacio, luz y nutrientes necesarios para su desarrollo.

Olvera-Vargas y Figueroa-Rangel (2012) sugieren que la competencia entre los individuos de la población es simétrica entre todos los tamaños diamétricos. Cuando los individuos presentan en su mayoría tamaños diamétricos o relativamente similares, como es el caso de este estudio en la parcela dos del área de bosque intervenido (Cuadro 4) en la especie de Q. sapotifolia la competencia se vuelve asimétrica y se presenta un efecto negativo sobre clases diamétricas inferiores, así mismo estos individuos conforme el tiempo crecen y envejecen dando lugar a competencia por luz solar, agua y nutrientes para su desarrollo.

El comportamiento de los tamaños diamétricos nos muestra que, en las parcelas de estudios, se encuentra un bosque maduro en desarrollo, encontrando individuos con diámetros de hasta $79 \mathrm{~cm}$ respectivamente. Sin embargo, haciendo uso de un barrenador, las edades de los árboles por parcelas indicaron árboles en un estimado de 25 años, presentándose un bosque coetáneo. Por otra parte, debido al aprovechamiento en las otras parcelas del área intervenida, hay disimilitudes en cuanto a su edad, ya que se han extraído los árboles de mayor edad y de mayor diámetro $(79 \mathrm{~cm}$ para Quercus), oscilando las edades entre los 13 y 18 años, presentándose un bosque joven probablemente producto de la regeneración natural, principalmente en la parcela dos con diámetros inferiores a los $30 \mathrm{~cm}$ (Quercusy Pinus)(Cuadro4).

Cuadro 4. Categoría diamétrica de especies en función a su diámetro por parcela de estudio, Reserva Santa Rosa, Estelí

\begin{tabular}{rccccc}
\hline Categorias diamétricas & Especie & $\mathrm{D}(\mathrm{cm}) \mathrm{P} 1 \mathrm{~Bq}$ & $\mathrm{D}(\mathrm{cm}) \mathrm{P} 2 \mathrm{~Bq}$ & $\mathrm{D}(\mathrm{cm}) \mathrm{P} 1 \mathrm{AI}$ & $\mathrm{D}(\mathrm{cm}) \mathrm{P} 2 \mathrm{AI}$ \\
\hline \multirow{5}{*}{$10-19.99 \mathrm{~cm}$} & Q. sapotifolia & 16 & 19.5 & - & $15.4,15.7,16.4,17.5$ \\
& P. maximinoi & - & - & - & $14.9,19,19.7$ \\
& Q. sapotifolia & $21,22,25,27$ & 29.5 & - & $21.8,22.4,23.2,26.5$ \\
$20-29.99 \mathrm{~cm}$ & P. maximinoi & 21.5 & - & - & 21.8 \\
& Q. sapotifolia & - & $34.5,36$ & $38.6,38.7$ & 31.6 \\
$30-39.99 \mathrm{~cm}$ & P. maximinoi & - & - & 39.5 & 31.9 \\
& Q. sapotifolia & - & 48 & 40,43 & 42.5 \\
$40-49.99 \mathrm{~cm}$ & P. maximinoi & 45,46 & - & $41,43.6,46.5$ & - \\
& Q. sapotifolia & - & - & - & - \\
$50-59.99 \mathrm{~cm}$ & P. maximinoi & - & - & 59.6 & - \\
& Q. sapotifolia & - & - & - & - \\
$60-69.99 \mathrm{~cm}$ & P. maximinoi & 66 & - & - & - \\
& Q. sapotifolia & - & - & 79 & - \\
$70-79.99 \mathrm{~cm}$ & P. maximinoi & - & 79 & 75.5 & 75.5 \\
\hline
\end{tabular}

D: Diámetro, P: Parcela, Bq: Bosque, AI: Área intervenida.

Cambios ambientales con énfasis en la vegetación a través de indicadores forestales. Los valores de IR, que incorpora la densidad relativa y el volumen relativo, presentaron un valor de $139.57 \%$ para Pinus maximinoi en la parcela dos del área de bosque intervenida, en particular en esta parcela se presentó el mayor número de esta especie con 9 individuos y $124.93 \%$ para Quercus sapotifolia en la parcela dos del área de bosque, con cinco individuos respectivamente (Cuadro 5). 
Cuadro 5. Índice de rendimiento de especies asociadas en la Reserva Santa Rosa, Estelí

\begin{tabular}{lcccc}
\hline \multicolumn{1}{c}{ Especie } & \multicolumn{4}{c}{ Índice de rendimiento (R) } \\
\hline & 1 & 2 & 1 & 2 \\
Parcela & (Bosque) & (Bosque) & (Intervenida) & (Intervenida \\
Quercus sapotifolia & 72.05 & 124.93 & 88.46 & 60.43 \\
Pinus maximinoi & 127.95 & 75.07 & 111.54 & 139.57 \\
\hline
\end{tabular}

Olvera-Vargas et al. (2010) establecieron métodos de ordenación indirecta y directa para investigar los patrones dominantes de la composición florística, y para determinar si la variabilidad ambiental medida explica los patrones de variación florística a partir del Índice de Rendimiento (IR). Así mismo, establecieron parámetros que fueron consideradas como superfluas para explicar patrones florísticos en la composición de especies de dosel, estas fueron altitud, inclinación de la pendiente, aspectos florísticos y topografía.

Sin embargo, estos parámetros evaluados de acuerdo a las condiciones del sitio demostraron que a medida que las variaciones altitudinales varían, la composición de las especies bajo el dosel cambia, pero no se reflejan cambios sustanciales a partir de los otros parámetros en cuestión.

La estructura reflejada en el estudio demuestra que los patrones florísticos en cuanto a su composición demuestra que los valores altos en el IR en la parcela dos del bosque intervenido, se debe a que existe una etapa sucesional inicial de esta especie, debido al proceso de regeneración natural, este proceso se lleva a cabo debido al proceso de aprovechamiento forestal de Pinus, los diámetros estudiados varían por debajo de los $30 \mathrm{~cm}$, este comportamiento radica también a que los factores abióticos tales como la luz solar, el agua y los nutrientes están disponibles para los individuos presentes en la asociación.

Daehler (2003) sugiere que el IR también puede ser interpretado como un indicador de la influencia de la calidad del sitio sobre la heterogeneidad estructural observada entre asociaciones, ya que comprende características de los árboles como la densidad y el volumen que son afectadas por condiciones de sitio. Las especies arbóreas solo se limitan a dos especies (Quercus-Pinus) los valores diferenciados en cuanto al IR en estos sitios se debe a que existe una heterogeneidad en cuanto a sus diámetros y de igualmente a sus alturas correspondientes.

En relación con el gradiente de altitud, esta es la variable ambiental más importante para determinar patrones de composición florística en los bosques dominados por Quercus, que se encuentran bajo el dosel bajo y medio. Estudios similares realizados en otras latitudes también han encontrado que la altitud (o factores relacionados con la altitud, como la humedad) es el determinante más importante del cambio en la composición florística, dando lugar a la zonación florística (Hemp, 2006). La abundancia de especies, la densidad de individuos, área basal y volumen de fustes fueron también determinados además por otros factores como la precipitación y la topografía a una altitud de $1200 \mathrm{msnm}$. En las tierras de Chiapas, México las diferencias florísticas entre las asociaciones vegetales encontradas fueron relacionadas con la precipitación en la época lluviosa y con las temperaturas máximas durante la época seca, presentándose mayor diversidad conforme aumenta la precipitación y se reduce la temperatura (Cayuela et al., 2006).

\section{CONCLUSIONES}

La caracterización florística y estructural del bosque de pino dominado por encino, en la Reserva Santa Rosa reportó 13 familias, 14 géneros y 19 especies arbóreas, herbáceas y arbustivas que fueron influenciadas por gradientes ambientales como la altitud, temperatura y precipitación; este comportamiento influenciado evidenció familias generalistas y otras más selectivas a nivel del dosel medio y bajo, además de encontrar variaciones en cuanto al diámetro de dichas especies. La dominancia del dosel se identificó por Q. sapotifolia en asocio con P. maximinoi.

Los datos estadísticos mostraron que la frecuencia diamétrica y las densidades de las especies de Pinus y Quercus no mostraron diferencias significativas en el rodal. Se reportan dos similitudes vegetales con base a la dominancia del Quercus, la mayoría de las asociaciones presentadas en cuanto al número de familias se encontró en el dosel medio y bajo del bosque. El coeficiente de Gini reflejó que las áreas intervenidas fueron las más desiguales con tamaños diamétricos heterogéneos debido al proceso de regeneración y la competencia entre los individuos.

Los valores de IR más altos en cuanto a su densidad y volumen fueron claramente definidos en el área del bosque, particularmente al presentar mayor número de individuos. La estructura del sitio demostró que los patrones de distribución estuvieron influenciados principalmente por la altitud, precipitación y topografía respectivamente.

\section{AGRADECIMIENTO}

Agradecemos la valiosa colaboración y aporte a los Fondos Concursables de la Dirección de Investigación, Extensión y Posgrado (DIEP), por los fondos aprobados para esta investigación; al Lic. Benito Quezada por su aporte en la identificación de especies herbáceas y arbustivas y al Sr. Eriberto Cerrato, dueño de la Reserva Santa Rosa, por aceptar la investigación en su propiedad. 


\section{REFERENCIAS BIBLIOGRÁFICAS}

Cayuela, L., Golicher, D., Rey-Benayas, J. M., González-Espinoza, M. y Ramírez-Marcial, N. (2006). Fragmentation, disturbance and tree diversity conservation in tropical montane forests. Journal of Applied Ecology, (43), 1172-1181. https://doi.org/10.1111/j.13652664.2006.01217.x

Cornide Rivas, J., Pou Ametler, M., Suari Andreu, L. y Solé Ruiz, L. (2008). Proyecto ecoturístico integrado en la Reserva Natural El Tisey-La Estanzuela [Tesis de pregrado, Universitat Autònoma de Barcelona (UAB)]. Repositorio Institucional UNAN-Managua. https://repositorio.unan.edu.ni/1834/1/TES\%201034.pdf

Challenger, A. y Caballero, J. (1998). Utilización y conservación de los ecosistemas terrestres de México. Pasado, presente y futuro. Comisión Nacional para el conocimiento y Uso de la biodiversidad.

Daehler, C. C. (2003). Performance comparisons of co-occurring native and alien invasive plants: Implications for conservation and restoration. Annual Review of Ecology and Systematics, (34), 183-211. https://www.jstor.org/stable/30033774

Dixon, P. M. (2001). The Bootstrap and the Jackknife. Describing the precision of ecological indices. En: S.M. Scheiner y J. Gurevitch (Eds.), Design and Analysis of Ecological Experiments (pp.267-288). Oxford University Press.

Encina Domínguez, J. A., Zárate Lupercio, A., Estrada Castillón E., Valdés Reyna, J. y Villarreal Quintanilla, J. Á. (2009). Composición y aspectos estructurales de los bosques de encino de la sierra de Zapalinamé, Coahuila, México. Acta Botánica Mexicana, (86), 71108. http://www.scielo.org.mx/pdf/abm/n86/n86a4.pdf

Galindo-Jaimes, L., González-Espinosa, M., Quintana- Ascencio, P. y García-Barrios, L. (2002). Tree composition and structure in disturbed stands with varying dominance by Pinus spp. in the highlands of Chiapas, México. Plant Ecology, (162), 259-272. https:// doi.org/10.1023/A:1020309004233

Gentry, A. K. (1996). A field guide to the families and genera of woody plants of Northwest South America (Colombia, Ecuador, Perú) with supplementary notes in herbaceous taxa. University of Chicago Press.

Hemp, A. (2006). Continuum or zonation? Altitudinal gradients in the forest vegetation of Mt. Kilimanjaro. Plant Ecology, (184), 27-42. https://doi.org/10.1007/s11258-005-9049-4

Holdridge, L. y Poveda, L. (1997). Árboles de Costa Rica Volumen I: Palmas y otras monocotiledóneas arbóreas y árboles con hojas compuestas o lobuladas. Centro Científico Tropical.

Husch, B., Miller, C. I. y Beers, T. W. (1982). Forest Mensuration. Wiley.

Jardim, A., Killeen, T. y Fuentes, A. (2003). Guía de los árboles y arbustos del Bosque Seco Chiquitano, Bolivia. Editorial FAN.

Johnson, P. S., Shifley, S. R. y Rogers, R. (2002). The Ecology and Silviculture of Oaks. CABI Publishing

Kappelle, M. (2006). Neotropical Montane Oak Forests: Over view and Outlook. En M. Kappelle (Ed.), Ecology and Conservation of Neo tropical Montane Oak Forests (pp.449-467). Springer-Verlag.

Keller, R. (2004). Identification of tropical woody plants in the absence of flowers (2da ed.). Birkhauser.

Killen, T., Garcia, E. y Beck, S. (1998). Guía de árboles de Bolivia. Herbario nacional de Bolivia; Missouri Botanical Garden. https:// rb.gy/fmoca6

Koleff, P. y Urquiza-Haas, T. (2011). Planeación para la conservación de la biodiversidad terrestre en México: retos en un país megadiverso. Comisión Nacional para el Conocimiento y Uso de la Biodiversidad; Comisión Nacional de Áreas Naturales Protegidas. https://rb.gy/3w1phw

Luna-Vega, I., Alcántara-Ayala, O., Ruíz-Jiménez, C. A. y Contreras-Medina, R. (2006). Composition and structure of humid montane oak forests at different sites in Central and Eastern Mexico. En M. Kappelle (Ed.), Ecology and Conservation of Neo tropical Montane Oak Forests (pp. 102-112). Springer-Verlag.

Ministerio de Recurso Naturales y del Ambiente. (2007). Estado del ambiente de Nicaragua, III informe GEO 2003-2006. http://www. bio-nica.info/Biblioteca/Marena2007IIInformeEstadoAmbAP.pdf

Metsaranta, J. M. y Lieffers, V. J. (2008). Inequality of size and size increment in Pinus banksiana in relation to stand dynamics and annual growth rate. Annals of Botany, 101(4), 561-571. https://www.jstor.org/stable/43576061

Morrone, J. J. (2005). Hacia una síntesis biogeográfica de México. Revista Mexicana de Biodiversidad, 76(2), 207-252. https://doi. org/10.22201/ib.20078706e.2005.002.303

Olvera-Vargas, M., Figueroa-Rangel, B. L. y Vázquez-López, J. M. (2010). Is there environmental differentiation in the Quercusdominated forests of west-central Mexico?. Plant Ecology, (211), 321-335. https://doi.org/10.1007/s11258-010-9792-z

Olvera-Vargas, M. y Figueroa-Rangel, B. (2012). Caracterización estructural de bosques montanos dominados por encino en el centrooccidente de México. Ecosistemas, 21(1-2). 74-84. https://www.revistaecosistemas.net/index.php/ecosistemas/article/view/28

Olvera-Vargas, M., Figueroa-Rangel, B. L. y Vázquez-López, J. M. (2015). Tree mortality and recruiment in heterogeneous stands of sub-tropical mixed-oak forest in west-central México. Interciencia, 40 (6), 233-240. https://www.redalyc.org/articulo. oa? id=33935906002

Rocha-Loredo, A. G., Ramírez-Marcial, N. y González-Espinosa, M. (2010). Riqueza y diversidad de árboles del bosque tropical caducifolio en la Depresión Central de Chiapas. Boletín de la Sociedad Botánica de México, (87), 89-103. https://doi.org/10.17129/ botsci.313 


\section{REFERENCIAS BIBLIOGRÁFICAS}

Rubio-Licona, L. E., Romero-Rangel, S., y Rojas-Zenteno, E. C. (2011). Estructura y composición florística de dos comunidades con presencia de Quercus (Fagaceae) en el Estado de México. Revista Chapingo. Serie Ciencias Forestales y del Ambiente, 17(1), 7790. https://doi.org/10.5154/r.rchscfa.2010.03.014

Rzedowski, J. (1996). Análisis preliminar de la flora vascular de los bosques mesófilos de montaña de México. Acta botánica mexicana, (35), 25-44. https://doi.org/10.21829/abm35.1996.955

Siles G., P., Talavera A., P., Andino Rugama, F., Alaniz, L., y Ortiz González, W. (2017). Composición florística, estructura y biomasa de los bosques de pino-encino en la reserva Santa Rosa, Tisey, Estelí, Nicaragua. Revista de Biología Tropical, 65(2), 763-775. https://doi.org/10.15517/rbt.v65i2.22928

Ulloa, C. y Jorgensen, P. (1993). Árboles y arbustos de los Andes del Ecuador. Pontificia Universidad Católica del Ecuador.

Wenger, K. F. (1984). Forestry Handbook (2da ed.). Wiley-Interscience.

Zamora Villalobos, N. (2000). Árboles de la mosquitia hondureña: descripción de 150 especies. Centro Agronómico Tropical de Investigación y Enseñanza. http://orton.catie.ac.cr/repdoc/A7027e/A7027e.pdf

Zamora Villalobos, N., Jiménez Madrigal, Q. y Poveda Álvarez, L. (2000). Árboles de Costa Rica. Volumen II. Instituto Nacional de Biodiversidad (INBio).

Zamora Villalobos, N., Jiménez Madrigal, Q. y Poveda Álvarez, L. (2004). Árboles de Costa Rica. Volumen III. Instituto Nacional de Biodiversidad (INBio).

Zamora, N. y Pennington, T. (2001). Guabas y guajiniquiles de Costa Rica (Inga spp.). Instituto Nacional de Biodiversidad (INBio). 